\title{
On the myths in hydrology and in other earth sciences
}

\author{
Serguei Dobrovolski* ${ }^{*}$ Mariia Istomina, and Irina Lebedeva \\ Water Problems Institute RAS, Gubkina 3, 119333, Moscow, Russia
}

\begin{abstract}
Following scientific myths, which are widely spread in hydrology and in other earth sciences, are discussed. (1) The total water mass on land surface statistically significantly diminishes during last century. (2) Many time series of annual river runoff demonstrate nonstationary character. (3) The first-order Markov chain is a dominating model in the stochastic description of the long time series of annual runoff. (4) Because of the global heating, annual discharges of the northern rivers will inevitably grow during the $21^{\text {st }}$ century. (5) The main contribution to uncertainty of the forecast of the runoff through the end of the $21^{\text {st }}$ century is made by uncertainty in scenarios of emission of greenhouse gases and divergences in results of modeling of the climatic system by GCMs. (6) The most shocking myth: the idea of the deterministic positive trend within the mean global temperature during last 100 years.
\end{abstract}

\section{Land water mass}

It is usually supposed that eustatic changes of the global mean sea level (GMSL) reflect changes in the water mass on the land (lakes, glaciers, snow, etc.). In turn, it is believed that GMSL (see, for instance, Fig. 1) contains statistically significant positive trend.

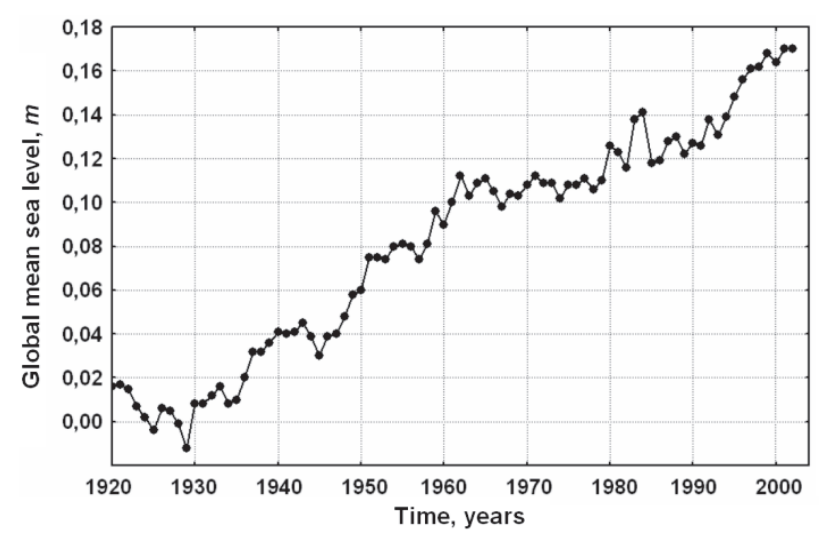

Fig. 1. Eustatic changes of the global mean sea level [1].

\footnotetext{
* Corresponding author: sgdo@bk.ru
} 
However, accurate analysis using applied methods of the random functions theory (through solving the characteristic autoregression equation [2]) shows that time series in Fig. 1 is satisfactorily described by the Wiener discrete process model:

$$
H(t)=H(t-1)+a(t)
$$

where $H$ - GMSL; $t$ - time in years; $a$ - normally distributed random value with zero mathematical expectation.

\section{Stationarity or nonstationarity of the river runoff time series?}

The idea of a nonstationary character of annual river runoff during last decades, presumably due to the global heating, is widely spread. Nevertheless, our analysis of about 1,000 time series of annual discharges with the length more than 40 years, using our new system of estimations of stochastic models' parameters [3], demonstrates following, more sophisticated pattern.

The percentage of nonstationary, with the respect to the mathematical expectation (i.e., with statistically significant monotonous trends), time series of the annual runoff of the rivers of main type of feeding (not regulated by reservoirs and lakes) is only $3.6 \%$. Appropriate value for the rivers of all types of feeding is $6,0 \%$.

However, the percentage of time series of minimal runoff, i.e. monthly runoff of the minimal discharge, is much higher and represents $17.3 \%$ for the rivers of main type and $21,4 \%$ for all analysed rivers. It is important to note, that even in these cases of the monotonous-type nonstationarities usually also manifest the effect of an intermittent nonstationarity. The latter effect means that the whole series is composed of a set of segments with statistically significant trend of different signs and of stationary segments.

\section{Stochastic (autoregression) models of annual runoff series}

It is usually assumed that the first order Markov process model (other terms: "first-order autoregression model", "simple Markov chain") satisfactorily describes most time series of annual river runoff values. We studied this problem introducing a new criteria for the choice of the order $M$ of an autoregression model fitting a time series.

Point is, existing criteria - Akaike criterion, Information Akaike criterion, ShwartzRissanen criterion, Parzen criterion, Hennan-Quinn criterion, and others - strongly depend on the length of a time series. A variant of the Akaike criterion, which practically does not depend on the length of a series, was proposed in [3]. Recall that technically the needed effect is obtained using multiplying the Akaike criterion for the zero order of autoregresssion by coefficient:

$$
K_{A C}=a_{1}+\frac{a_{2}+a_{3} r_{1}}{N}+\frac{a_{4}}{N^{2}}+r_{1}\left(a_{5}+a_{6} r_{1}\right)
$$

where $r_{1}$ is a sample estimation of correlation between neighbouring members of the series; $N$ - the length of the series in years; constants $a$ are specially chosen for different diapasons of $r_{1}$ and $N$ in order to artificially fit the criterion to a uniform length $N_{\mathrm{U}}$ of a series. In our case $N_{\mathrm{U}}$ was equal to 40 years, which is the mean length of the river runoff series in our archive.

In Fig. 2 results of calculations of $M$ for the most numerous gauges, at the rivers of the main type (not regulated by lakes and dams) are shown. The results are obtained in two variants - for the initial series and for series recalculated into the sample values of the 
normally distributed random numbers. It is evident that both variants demonstrate absolute predominance of the zero order of autoregression models: their numbers exceed not only the numbers of the first order models, but the number of all models with $M>0$.

Thus, the hypothesis of a universal applicability of the "simple Markov chain" (first order autoregression model) is rejected on the basis of accurate analysis of almost 1,000 series of the annual discharges of the rivers of the world of main type.

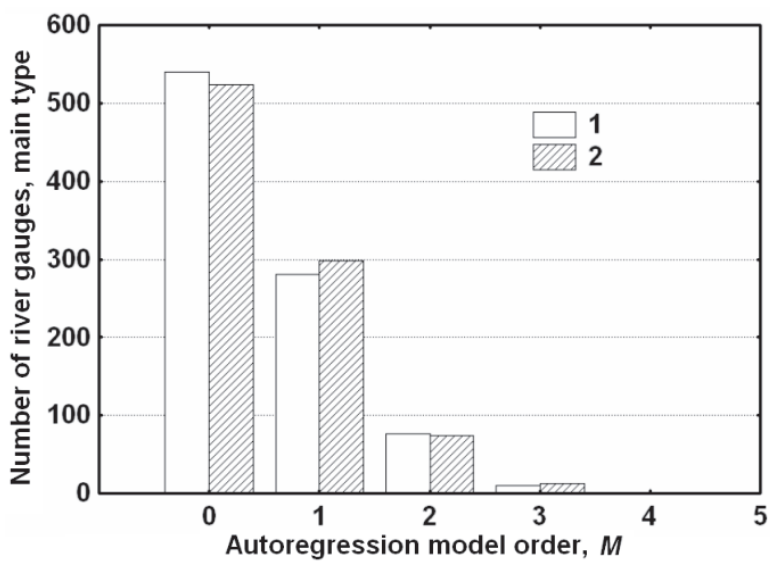

Fig. 2. Histogram of the orders $M$ of autoregression models for the description of time series of annual discharges at the river gauges. Rivers of the main type - not regulated by lakes and reservoirs. 1 - initial series, 2 - time series, recalculated into the series of sample values of the Gaussian random numbers.

\section{Forecasting the river runoff: scenarios for the $\mathrm{XXI}$ century}

It is supposed in many works that the annual runoff of the rivers of the northern slopes of Eurasia and North America will inevitably rise during the $21^{\text {st }}$ century. These forecasts, at best, take into consideration only two types of uncertainties of such predictions: due to the uncertainties of the future emissions of the greenhouse gases into the atmosphere (IPCC scenarios are usually applied), and due to divergences between different climate models, GCMs.

In our investigations of the problem we added following improvements. (1) We took 6 IPCC scenarios and added to them a scenario elaborated by the team of the Moscow Power Engineering Institute. (2) We took into consideration two additional types of uncertainties: due to the errors of calculating mean runoff during the test period of instrumental observations, and due to possible natural changes of the global temperatures. In this way we used the results of prognostic experiments on 21 IPCC GCMs with improved land hydrology blocks, and our methods of calculating errors in the "natural" mean river runoff and errors due to possible natural changes in the global temperatures described in the $6^{\text {th }}$ section of this paper. The detailed description of our methods and formulas is given in [4].

Fig. 3 shows our forecast of the annual runoff of the Lena river through the year 2100 . Here the Moscow Power Engineering Institute scenario of the greenhouse gases emission is used. We prefer this scenario because based on it the forecasts of the greenhouse concentration in the atmosphere and of the global temperature, made in the late 1980s, demonstrate better coincidence with real changes up to $2010 \mathrm{~s}$. The figure shows that considerable growth of discharges during the 21 st century is as possible as the absence of changes. 


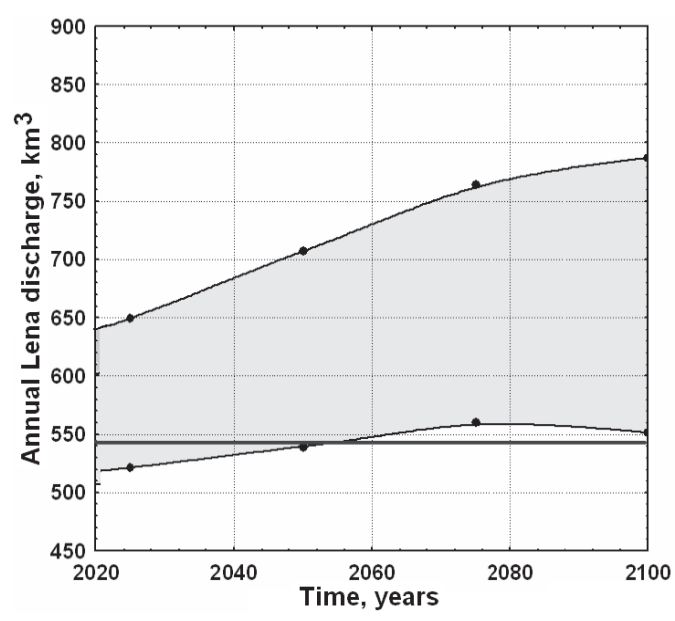

Fig. 3. Forecasting of the mean annual Lena runoff. Horizontal line denotes mean runoff for the instrumental period. Future changes in the mathematical expectation of the runoff plus-minus mean square root error of its estimation are denoted by grey color.

\section{Sources of uncertainties in future runoff changes}

While forecasting possible changes in the river runoff during the $21^{\text {st }}$ century, researchers use to consider only two sources of uncertainties: those due to differences between scenarios of the greenhouse gases emissions into the atmosphere, and uncertainties due to imperfection of GCMs. However, Fig. 4 demonstrates the importance of two other types of uncertainties.

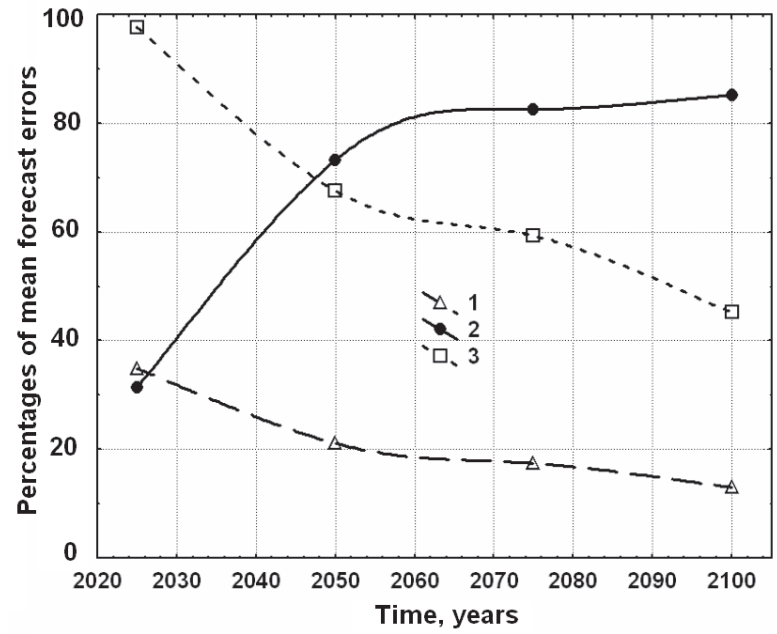

Fig. 4. Relations between mean square root errors of the Lena annual discharges forecast due to different sources of uncertainties, in \%. 1 - due to the errors of estimating mean runoff during the test period of instrumental observations; 2 - due to the differences between 6 IPCC scenarios of greenhouse emissions; 3 - due to imperfection of GCMs. Mean square errors due to possible natural changes in global climate are supposed to be equal to $100 \%$. 
It is seen that uncertainties due to possible natural variations in global climate are always larger than other types of uncertainties. The same is true for other rivers of the northern slopes of Eurasia and of North America.

\section{Global temperature rise: greenhouse effect or random walk?}

Mean global temperature (GT- see Fig. 5) is the main parameter in climatology, hydrology, etc. Most researchers, especially those who write the IPCC reports, insist without hesitations that the GT general growth during last century is due to the greenhouse effect.

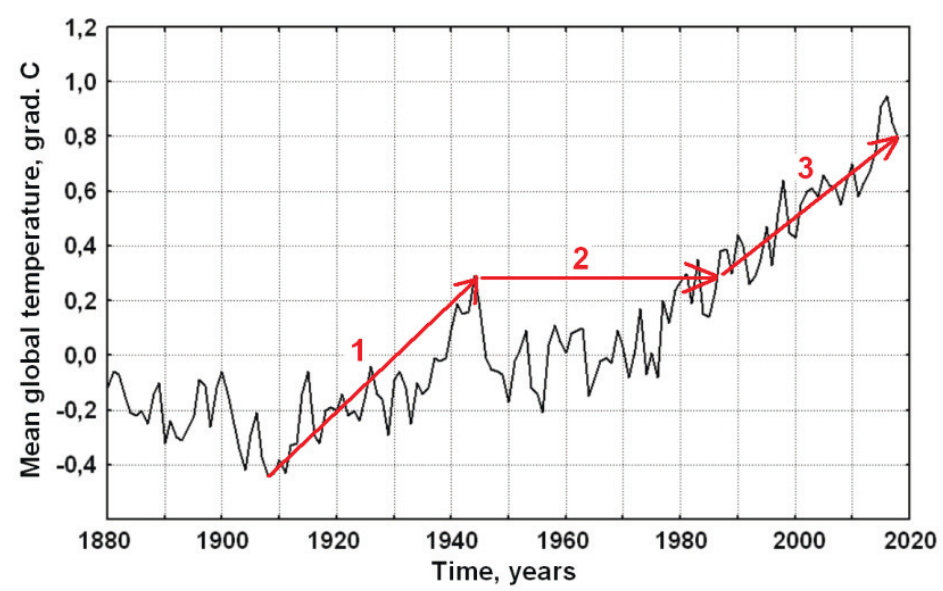

Fig. 5. Mean global annual air temperatures. After [5]. Numbers $1-3$ correspond to the different segments of the series denoted by the arrows. See explanations in text.

However, implementation of the theory of random functions and its application, the Maximum Entropy Method, shows the following. Solving the characteristic autoregression equation gives a unique root, which is located practically on the unit circle on the complex plain. It means that the nonstationary sequence shown in Fig. 5 might be described using the Wiener discrete model (see eq. 1), and the first increments of the sequence (Fig. 6) might be a stationary autoregression sequence with zero order $M$, i.e., the white noise.

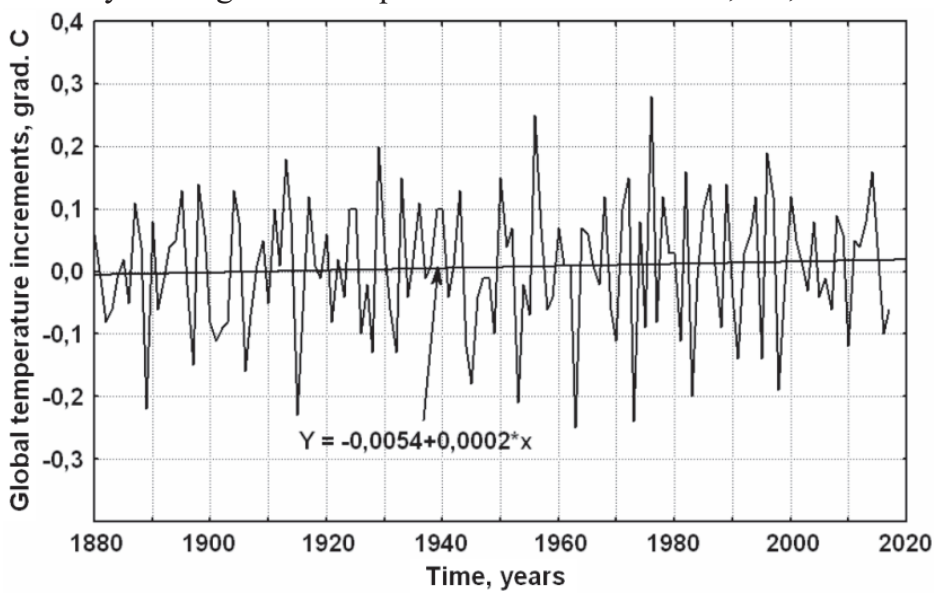

Fig. 6. Year-to-year increments of the global mean temperature series shown in Fig. 5. 
Indeed, analysis of the time series of the first increments confirms this hypothesis. Namely, our system of estimating the order $\mathrm{M}$ gives zero value, and the mean value of increments for the period $1881-2018$ is very close to zero - equal to $0.00659{ }^{\circ} \mathrm{C} /$ year (and this mean value grows very slowly in time - see solid line in Fig. 6), which is less than standard error of this estimation, $0.00751{ }^{\circ} \mathrm{C} /$ year. Thus, the random walk model perfectly fits changes in the global mean temperatures, i.e., the letter does not include any deterministic monotonous trend. The same is true if we take into consideration only the period of "early" heating (mid 1900s - mid 1940s) or only the period of last heating (from 1970s).

By the way: formally, the series in Fig. 5 can be considered as a manifestation of an effect of so-called "intermittent nonstationarity" within the sequence - stationary segment 2 is located between nonstationary segments 1 and 3. It is interesting that the effect of intermittent nonstationarity can be found within time series of different hydrometeorological parameters: drought index SPEI [6], changes in the annual discharges of rivers regulated by lakes and reservoirs [7], and some others.

This study was carried out under the subjects no. 0147-2019-0003 (state registration no. AAAA-A18118022090105-5) and no. 0147-2019-0001 (state registration no. AAAA-A18-118022090056-0) of the Governmental Order to Water Problems Institute, Russian Academy of Sciences, and was supported by the Russian Foundation for Basic Research, project no. 19-05-00375.

\section{References}

1. IPCC. Special report on the ocean and cryosphere in a changing climate. https://www.ipcc.ch/svoce/home (2019)

2. S.G. Dobrovolski, Stochastic climate theory (Springer, Heidelberg, 2000)

3. S.G. Dobrovolski. Global'nye izmeneniya rechnogo stoka (Global changes in the river runoff) (Geos, Moscow, 2011)

4. S.G. Dobrovolski. Global'naya gidrologiya (Global hydrology) (Geos, Moscow, 2017)

5. NOAA. 4th Warmest Year. https://www.wunderground.com/cat6/Earth-Had-Its-4thWarmest-Year-Record-2018-Say-NOAA-and-NASA (2019)

6. S.M. Vicente-Serrano, S. Beguería, J.I. López-Moreno, J. of Clim., 23, 1696-1718 (2010)

7. S.G. Dobrovolski, I.P. Lebedeva, M.N. Istomina, I.V. Solomonova, Wat. Res., 47, 1-12 (2020) 